\title{
Preparation of a Cu Surface with the Hierarchical Structure of a Lotus Leaf via Electroplating and Its Superhydrophobicity
}

\author{
Kai Chen ${ }^{1,{ }^{* 1}}$, Tianchi Wang ${ }^{1, * 2}$, Yizhuo Zhao ${ }^{1}$, Zhe Zhou ${ }^{1}$, Ziheng Liu ${ }^{1}$, Jian Kong ${ }^{1}$ and Hongfu Xiang ${ }^{2}$ \\ ${ }^{1}$ School of Materials Science and Engineering, Nanjing University of Science and Technology, Nanjing 210094, China \\ ${ }^{2}$ School of Materials Science and Engineering, Jiangsu University of Science and Technology, zhenjiang 212003, China
}

\begin{abstract}
A lotus leaf exhibits excellent superhydrophobic properties because of its hierarchical microstructure and a waxy hydrophobic material covering its surface. Herein, the lotus leaf was used as a template and a copper surface layer with a microstructure similar to the lotus leaf was prepared by electroplating. Experimental results show that the plating samples saved micron-sized structure of the lotus leaf, such as papillae. At the same time, owing to the specificity of the electroplating method, smaller grains were formed as the second-level structure on the papillae, forming a hierarchical structure that makes it possible to obtain a superhydrophobic surface. The plated copper surface exhibited excellent hydrophobic properties with a contact angle of $160^{\circ}$, which is higher than that of the lotus leaf $\left(157^{\circ}\right)$ and also much higher than that of smooth copper covered with an identical fluroalkyl silane $\left(112^{\circ}\right)$. This affords a novel idea for preparing of superhydrophobic surfaces. [doi:10.2320/matertrans.M2017139]
\end{abstract}

(Received April 27, 2017; Accepted June 5, 2017; Published July 7, 2017)

Keywords: electroplating, copper, wetting, biotemplate

\section{Introduction}

Copper plays an important role in our daily life and is widely used in industry ${ }^{1,2)}$. When exposed to air for a long time, copper products ultimately get oxidized and corroded as dust and moisture adhere to them. At the same time, copper has a high surface energy, which makes it easier for it to adsorb water droplets in refrigeration equipment and outdoor coolers ${ }^{3)}$. This results in the degradation of the electrical and mechanical properties of the copper material and reduces its service life. Therefore, it is lucrative to develop copper materials that possess a superhydrophobic surface, which may be expected to greatly reduce the possibility of dust, liquids, and other foreign impurities adsorbing on its surface $^{3)}$. For example, the pipeline made of the superhydrophobic copper can prevent a pipeline from corrosion of water and reduce the transportation resistance ${ }^{4)}$. Electrical equipments expose to cold environments that made of superhydrophobic copper can be protected against freezing and frost.

In recent years, several methods have been reported for the preparation of copper materials with a superhydrophobic surface. To achieve this objective, a rough surface structure is constructed and treated with a low surface energy material. In this process, the construction of an appropriately rough surface structure is crucial $^{5-8)}$. A rough copper surface can be obtained by several methods such as chemical oxidation $^{9,10)}$, chemical etching ${ }^{11,12)}$, physical etching ${ }^{13)}$, physical and chemical film deposition ${ }^{14)}$, and hydrothermal synthe$\operatorname{sis}^{15)}$.

Inspired by the unique structures and functions of plants and animals, researchers have proposed the morph-genetic materials that employed the structures of living things as templates. Morph-genetic materials are prepared by preserving the structures of living things and using physical and chemical means to change their composition to meet the

\footnotetext{
${ }^{* 1}$ Graduate Student, Nanjing University of Science and Technology

${ }^{* 2}$ Corresponding author, E-mail: tianchiwang@aliyun.com
}

needs of the people. Owing to the complex structures of plants and animals, their morph-genetic materials exhibit unique properties. Therefore, many scholars have devoted their attention to this method for preparing novel materials.

A lotus leaf is a naturally superhydrophobic material because of its hierarchical surface structure. Nano-sized microvilli exist on the numerous papillae, and a waxy substance is also attached to its surface. Under the combined action of the structure and composition, the surface of the lotus leaf exhibits high hydrophobicity ${ }^{16}$. If the special structure of a lotus leaf is engraved onto the copper surface followed by treatment with a low-surface-energy material, the copper surface may be expected to attain superhydrophobic properties. However, it is important to resolve the issue of effectively copying the surface structure of a lotus leaf onto copper. The field of morph-genetic materials is inspiring in this regard. Therefore, in this study, a lotus leaf was used as a template for the preparation of superhydrophobic copper. This method involved electroplating the metal on the carbon derived from the lotus leaf, thus copying its unique hierarchical structure onto the copper surface.

\section{Experimental}

\subsection{Sample preparation}

In the first step, the fresh lotus leaves were dried in an oven for $24 \mathrm{~h}$ at $100^{\circ} \mathrm{C}$ to remove most of their moisture. The dried leaves were then sintered under an inert argon atmosphere by heating them at a rate of $5^{\circ} \mathrm{C} \mathrm{min}^{-1}$ to $200^{\circ} \mathrm{C}$ and at $2^{\circ} \mathrm{C} \mathrm{min}^{-1}$ to $800^{\circ} \mathrm{C}$. The final temperature of $800^{\circ} \mathrm{C}$ was maintained for $1 \mathrm{~h}$. In this manner, the organic material of the leaf was decomposed and carbonized during sintering and carbon with the structure of a lotus leaf was obtained. In the next step, the lotus leaf carbon was set as a cathode of an electrolytic cell and electroplating was carried out using copper, which was set as the anode. The current density, plating time, and concentration of the copper sulfate plating solution were $0.02 \mathrm{~A} \mathrm{~cm}^{-2}, 5 \mathrm{~min}$, and $0.3 \mathrm{~mol} \mathrm{~L}^{-1}$, respectively. After electroplating, a copper film with a structure 
similar to the surface of a lotus leaf was obtained. The copper film was then soaked into a mixed solution of fluoroalkyl silane (F-1060, $\left.\mathrm{C}_{8} \mathrm{~F}_{17} \mathrm{CH}_{2} \mathrm{CH}_{2} \mathrm{Si}\left(\mathrm{OC}_{2} \mathrm{H}_{5}\right)_{3}\right)$ and isopropyl alcohol with a volume ratio of 1:6 for five days. Finally, a superhydrophobic copper surface with a lotus leaf surface structure was obtained.

\subsection{Characterization}

The surface morphology of the samples was observed by FEI Quanta 250F field emission scanning electron microscopy. The crystal structures of the prepared samples were obtained using an X-ray diffractometer (XRD, D8 Advance, Germany). A contact angle meter (JC2000D2, Shanghai) was used to measure the static contact angle of the samples, using drops with volumes of $2 \mu \mathrm{L}$.

\section{Results and Discussion}

\subsection{XRD analysis}

Figures 1(a)-(c) are the full-scale images of a lotus leaf, carbon derived from the lotus leaf, and electroplated copper. It can be seen that the lotus leaf was completely converted to carbon after sintering without oxygen, and copper was successfully attached on the surface of the lotus leaf carbon via electroplating. It is also evident from the macro-structure that the shape of the lotus leaf was retained by carbon, and the texture of the leaf was also retained on carbon and electroplated copper. The XRD pattern of the electroplated copper is given in Fig. 1(d). It shows that the main components of the sample are copper and cuprous oxide. This observation suggests that the oxidation of copper occurs during the process of electroplating. Since a layer of copper was completely attached to the surface of the carbon template, no obvious diffraction peak corresponding to carbon could be seen in the XRD spectrum.

\subsection{SEM analysis}

Figures 2(a) and 2(b) show the microstructures of the lotus leaf. It can be seen that there are many micron-sized papillae on its surface, and numerous nano-sized microvilli are present on every papilla. Therefore, the lotus leaf exhib- its a hierarchical structure. Figures 2(c) and 2(d) show the surface morphology of the carbon derived from the lotus leaf. It can be seen that the carbon retained the micron-sized papillae structure of the lotus leaf. The organic material of the lotus leaf was decomposed during the carburizing sintering step and while a part of it was transformed into a gas and expelled, another part was transformed into carbon that maintained the leaf structure. However, the nano-sized microvilli structure had disappeared because the organic composition in microvilli decomposed completely and all the carbon turned into either carbon dioxide or carbon monoxide during the sintering process. Figures 2(e) and 2(f) show the surface morphology of copper electroplated on the carbon derived from lotus leaf. It is evident that this type of copper possesses a similar morphology as that of the lotus leaf. Although some of the microvilli structure of the lotus leaf was lost during sintering, small copper grains were formed on the papillae during the electroplating process. These small copper grains become the second-level structure on the surface, which makes up for the lost hierarchical structure of the lotus leaf during the sintering process. Figure 3 shows the schematic diagram of the changes in the surface microstructure of the samples at different stages of preparation, especially second-level structure on the papillae. It shows that there is a great number of nano-sized microvilli intersperse on the papilla of the fresh lotus leaf, and they disappear after sintering but a carbon frame left. Finally, the surface of carbon frame forms a layer of copper through plating. Furthermore, the copper grain replaces the microvilli and becomes the second-level structure.

\subsection{Superhydrophobic properties}

Figure 4 shows the surface contact angle of different materials and the sliding angle of plated copper. The contact angle of a natural lotus leaf is $153^{\circ}$, which is indicative of its superhydrophobic performance (as shown in Fig. 4(a) and 4(d)). This property arises because of its complex rough structure and the presence of a waxy substance on the surface. Pure copper is a hydrophilic material, even though it is polished into a low surface roughness. Its treatment with a low-surface-energy material is still insufficient for imparting
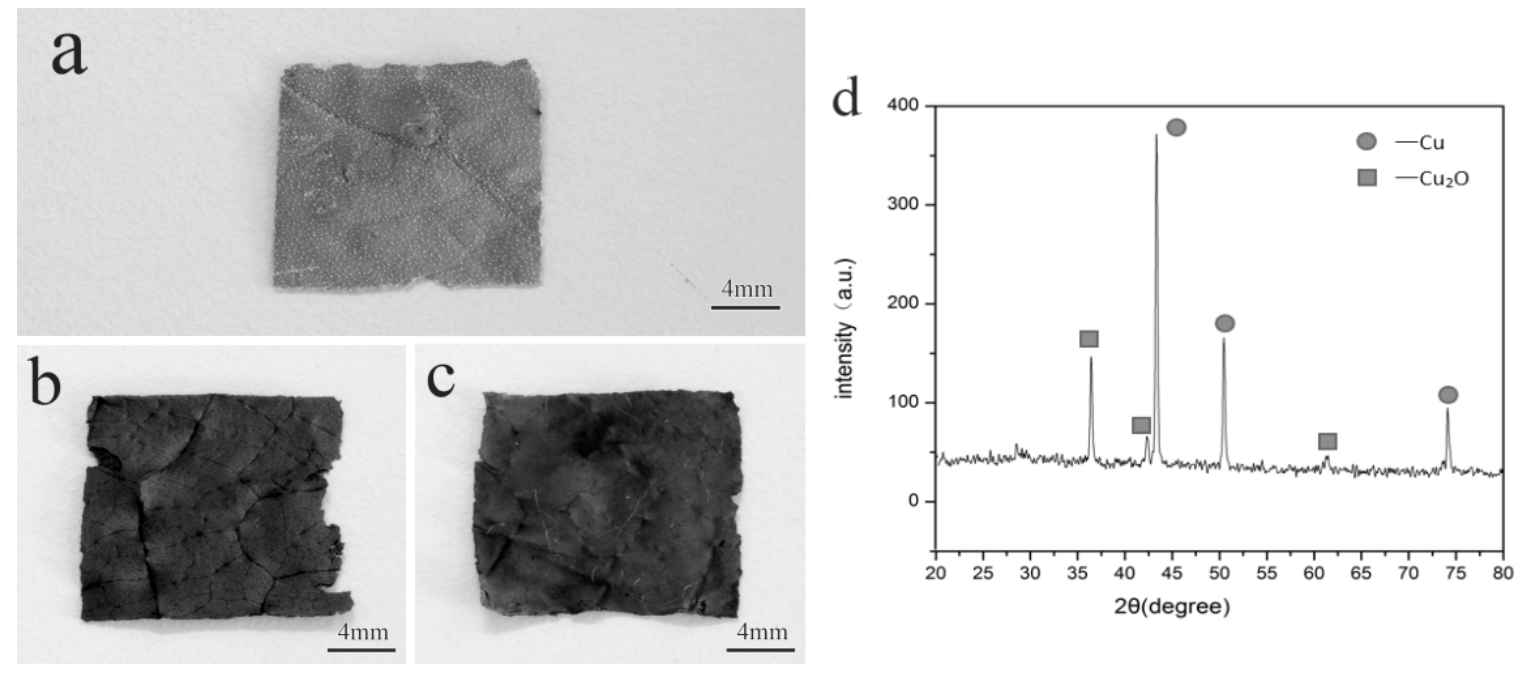

Fig. 1 (a) Lotus leaf, (b) carbon derived from lotus leaf, (c) electroplated copper, and (d) XRD pattern of electroplated copper. 

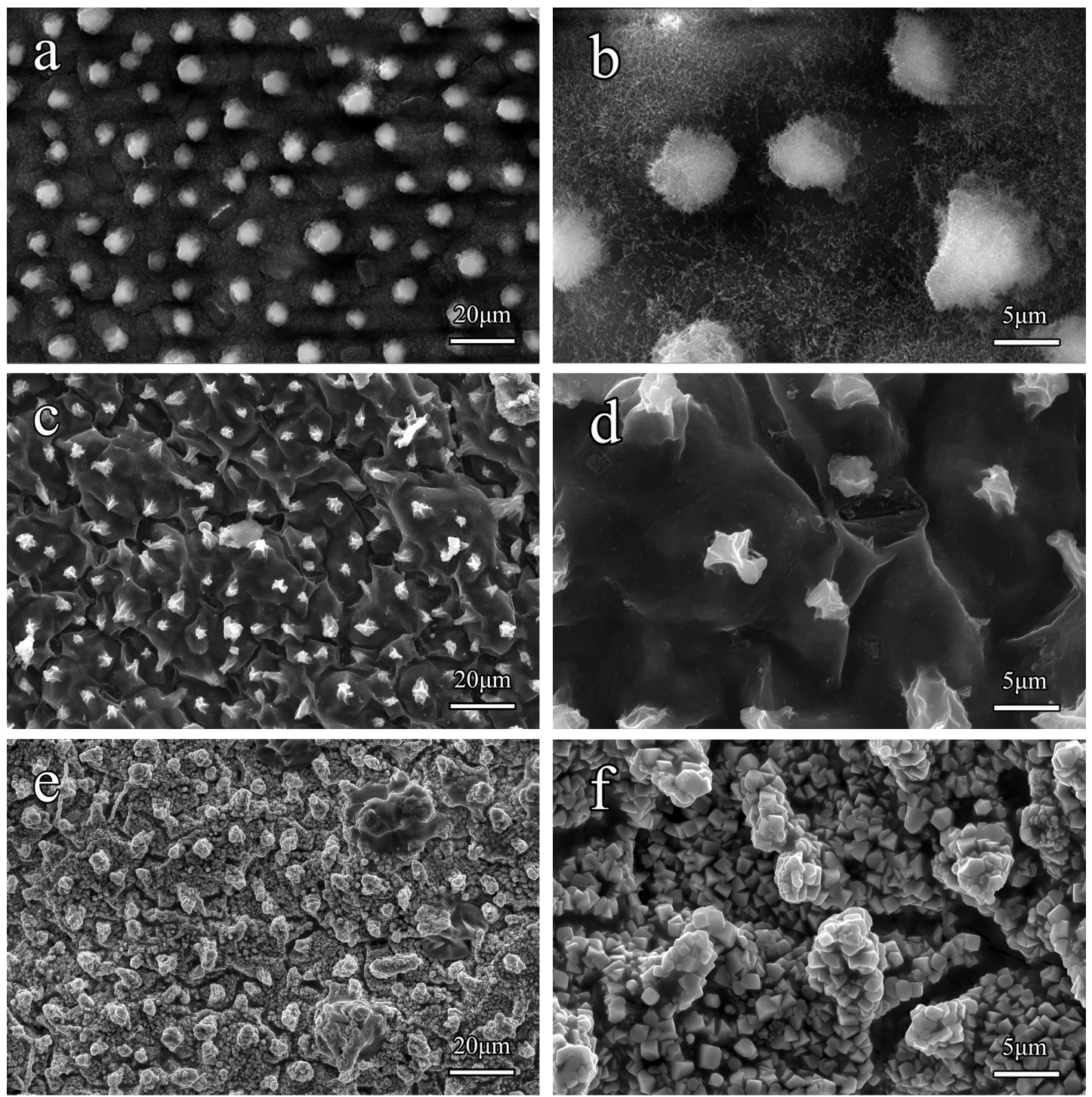

Fig. 2 SEM images of the sample surfaces obtained after different treatments. (a), (b) Lotus leaf, (c), (d) carbon derived from the lotus leaf, and (e), (f) electroplated copper.

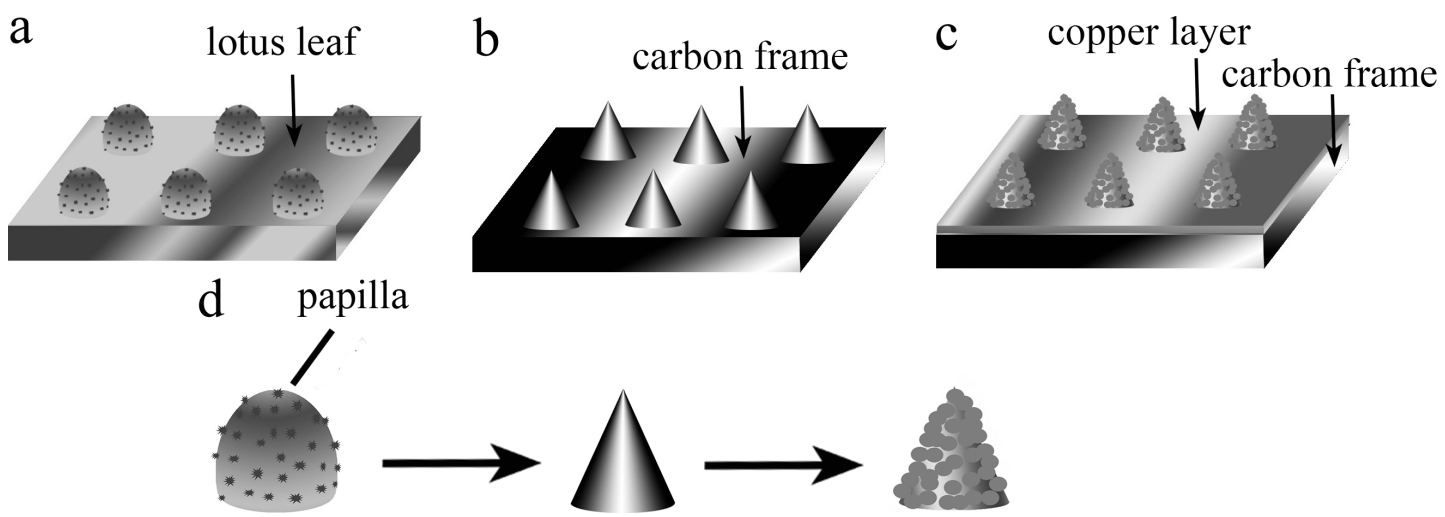

Fig. 3 A schematic diagram of the change in surface microstructure during the preparation processes. (a) Lotus leaf, (b) carbon derived from lotus leaf, (c) electroplated copper, and (d) the changing of a papilla.

hydrophobic property and the contact angle is only $112^{\circ}$ (as shown in Figs. 4(b) and 4(e)). However, the copper surface prepared by electroplating in this method showed superhydrophobic properties (as shown in Figs. 4(c) and 4(f)) once treated with fluroalkyl silane. Its contact angle is $160^{\circ}$, which is greater than that of the lotus leaf. Its sliding angle is $4.6^{\circ}$ (as shown in Figs. 4(g)), which shows excellent superhydrophobic properties with low adhesion. This is because the hierarchical structure of this type of copper surface is similar to that of the lotus leaf and that of the second-level 

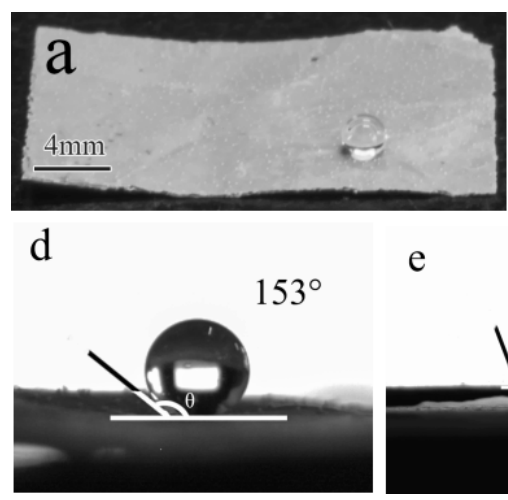

e
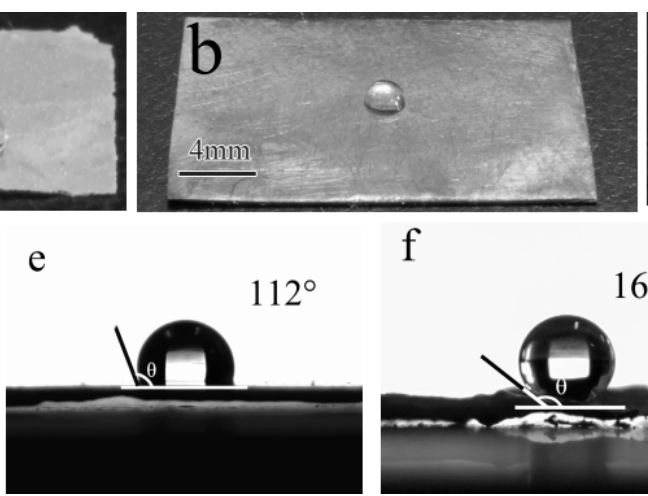

f

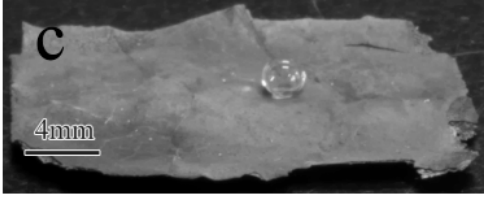

g
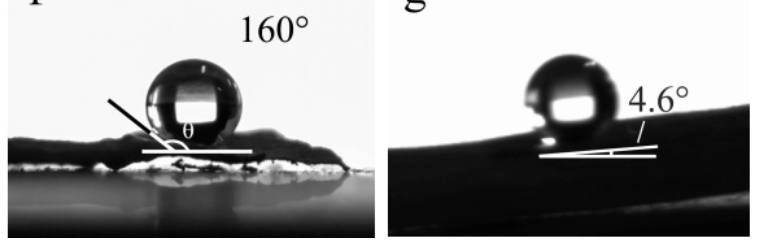

Fig. 4 Contact angles of (a), (d) lotus leaf, (b), (e) pure smooth copper, (c), (f) electroplated copper, and sliding angle of (g) electroplated copper.

grains is similar to the microvilli of the lotus leaf, ensuring its superhydrophobicity. These two surfaces depicted in Figs. 4(b) and 4(c) were also covered by fluroalkyl silane, but were found to have different hydrophobicities. This is because surface geometry exerts a significant effect on the wettability of a material. The leaf-like microstructure plays an important role in imparting superhydrophobic property to copper.

Since the material is hydrophobic, the mechanism of its hydrophobicity can be explained by the Cassie model ${ }^{17)}$. In this model, the equation that gives the contact angle is $\cos \theta^{*}=(1-f)(\cos \theta+1)-1$, where $\theta^{*}$ and $\theta$ represent the contact angles of water droplets on rough and smooth surfaces, respectively; $f$ corresponds to the percentage of the contact area between the water droplets and the entrapped air. According to the above results, the contact angle of the FAS-modified plated copper surface was $160^{\circ}$, while that of the FAS-modified smooth copper surface was $112^{\circ}$. The $f$ value obtained from the above equation for the plated copper surface was 0.904 . This indicates that only $9.6 \%$ of the water droplet is in contact with the plated copper substrate, whereas $90.4 \%$ of the water droplet is in contact with the entrapped air in the hierarchical structure. This shows that the water droplet and the substrate actually form point contacts, which is conducive for obtaining excellent hydrophobic property.

\section{Conclusion}

In this study, a lotus leaf was used as a template for the preparation of a superhydrophobic biomorphic copper surface by electroplating copper on the carbon derived from the lotus leaf. The plated copper formed a hierarchical structure that is similar to that of the lotus leaf and small copper grains were formed as the second-level structure on the papillae. This material exhibited an excellent superhydrophobic property after treatment with fluroalkyl silane. Its contact angle has been found to be $160^{\circ}$, which is higher than that of the lotus leaf, and its sliding angle is $4.6^{\circ}$, which shows excellent superhydrophobic properties. Therefore, it may be concluded that the method developed in the work can be utilized for preparing superhydrophobic copper materials and also provides a novel technique for the preparation of hydrophobic materials in general.

\section{Acknowledgements}

The authors wish to express thanks to the National Natural Science Foundation of China (51672131), the Fundamental Research Funds for the Central Universities (30916012203), and the Scientific Research Training Program of Undergraduates.

\section{REFERENCES}

1) E. Kim, M. Kim, J. Lee, K. Yoo and J. Jeong: Hydrometallurgy 100 (2010) 95-102.

2) Y. Chen, S. Chen, F. Yu, W. Sun, H. Zhu and Y. Yin: Surf. Interface Anal. 41 (2009) 872-877.

3) D.K. Sarkar and M. Farzaneh: J. Adhes. Sci. Technol. 23 (2009) 1215-1237.

4) A. Lafuma and D. Quéré: Nat. Mater. 2 (2003) 457.

5) S.H. Kim: J. Adhes. Sci. Technol. 22 (2008) 235-250.

6) B.N. Sahoo and B. Kandasubramanian: RSC Advances 4 (2014) 22053-22093.

7) T. Rezayi and M.H. Entezari: New J. Chem. 40 (2016) 2582-2591.

8) L. Zhang, N. Zhao and J. Xu: J. Adhes. Sci. Technol. 28 (2014) 769790.

9) R. Gao, J. Wang, X.F. Zhang, H.J. Yan, W.L. Yang, Q. Liu, M.L. Zhang, L.H. Liu and K. Takahashi: Colloids Surf. A Physicochem. Eng. Asp. 436 (2013) 906-911.

10) M.D. Pei, B. Wang, E. Li, X.H. Zhang, X.M. Song and H. Yan: Appl. Surf. Sci. 256 (2010) 5824-5827.

11) T. Darmanin, E.T. de Givenchy, S. Amigoni and F. Guittard: Adv. Mater. 25 (2013) 1378-1394.

12) Y. Huang, D.K. Sarkar and X. Grant Chen: Appl. Surf. Sci. 356 (2015) 1012-1024.

13) B. Wu, M. Zhou, J. Li, X. Ye, G. Li and L. Cai: Appl. Surf. Sci. 256 (2009) 61-66.

14) S. Rezaei, Manoucheri, R. Moradian and B. Pourabbas: Chemical Engineering Journal 252 (2014) 11-16.

15) A. Caldarelli, M. Raimondo, F. Veronesi, G. Boveri and G. Guarini: Surf. Coat. Tech. 276 (2015) 408-415.

16) A.T. Abdulhussein, G.K. Kannarpady, A.B. Wright, A.B. Wright, A. Ghosh and A.S. Biris: Appl. Surf. Sci. 384 (2016) 311-332.

17) A.B.D. Cassie and S. Baxter: Trans. Faraday Soc. 40 (1944) 546-551. 